\title{
Holocene changes in African vegetation: tradeoff between climate and water availability
}

C. Hély ${ }^{1}$, A-M. Lézine ${ }^{2}$, and the APD contributors

[1]\{ PALECO, CBAE, UMR 5059 CNRS-EPHE-Université Montpellier 2. Institut de Botanique, 163 rue Auguste Broussonnet, 34090 Montpellier, France\}

[2]\{ Sorbonne Universités (UPMC, Univ Paris 06)-CNRS-IRD-MNHN. LOCEAN laboratory, 4 place Jussieu, F-75005 Paris, France \} (1) 
Table S1. Characteristics of the West African sites from which fossil pollen assemblages have been extracted (African Pollen Database)

\begin{tabular}{|c|c|c|c|c|c|c|c|}
\hline $\begin{array}{l}\text { Site } \\
\text { ID }\end{array}$ & $\begin{array}{l}\text { Site } \\
\text { Name }\end{array}$ & $\begin{array}{l}\text { Latitude } \\
\qquad\left({ }^{\circ} \mathrm{N}\right)\end{array}$ & $\begin{array}{l}\text { Longitude } \\
\quad\left({ }^{\circ} \mathrm{E}\right)\end{array}$ & $\begin{array}{l}\text { Elevation } \\
\text { (m asl) }\end{array}$ & Country & $\begin{array}{c}\text { Time range } \\
\text { (yr BP) }\end{array}$ & References \\
\hline 1 & AMEKNI & 22.7850 & 5.5183 & 1000 & Algeria & 7663 & $\begin{array}{r}\text { (Guinet and Planque, } \\
\text { 1969) }\end{array}$ \\
\hline 2 & TAESSA & 23.1667 & 5.5167 & 2150 & Algeria & $4919-5661$ & $\begin{array}{r}\text { (Ballouche et al., 1995; } \\
\text { Thinon et al., 1996) }\end{array}$ \\
\hline 3 & BILGOY [ENNERI DIRENNAO] & 21.5183 & 17.1717 & 1135 & Chad & 8381 & (Schulz, 1973, 1980) \\
\hline 4 & KAMALA & 14.0333 & 16.3167 & 420 & Chad & 11784 & (Maley, 1981) \\
\hline 5 & KOUKA & 13.1000 & 15.6333 & 283 & Chad & 11002 & (Maley, 1981) \\
\hline 6 & MANDI & 13.3833 & 14.7500 & 276 & Chad & 11352 & (Maley, 1981) \\
\hline 7 & MOUSKORBE & 21.5833 & 18.8833 & 2600 & Chad & $7473-9443$ & (Maley, 1981) \\
\hline 8 & KA26 & 22.7333 & 16.6217 & $\mathrm{~N} / \mathrm{A}$ & Chad & 1780 & (Schulz, 1980) \\
\hline 9 & TARSO YEGA & 20.6667 & 17.5833 & 2200 & Chad & 7718 & (Maley, 1981) \\
\hline 10 & TJERI & 13.7333 & 16.5000 & 275 & Chad & $565-10094$ & (Maley, 1981, 2004) \\
\hline 11 & TJOLUMI & 21.5167 & 18.1333 & 1115 & Chad & 6647 & (Schulz, 1973, 1980) \\
\hline 12 & TROU AU NATRON & 21.0500 & 16.7500 & 1850 & Chad & 18188 & (Maley, 1981) \\
\hline 13 & BAIESAINT JEAN & 19.4707 & -16.3012 & 0 & Mauritania & $1778-3635$ & (Lézine, A.M. unpub. Data) \\
\hline 14 & CHEMCHANE 1 & 20.9333 & -12.2167 & 256 & Mauritania & $7590-9131$ & $\begin{array}{r}\text { (Lézine, 1987; Lézine et al., } \\
\text { 1990; Lézine, 1993) }\end{array}$ \\
\hline 15 & SEGUEDINE & 20.1667 & 12.7833 & 412 & Niger & 8777 & $\begin{array}{r}\text { (Baumhauer and Schulz, } \\
1984)\end{array}$ \\
\hline 16 & TERMIT & 16.2000 & 11.0667 & 450 & Niger & 9981 & (Schulz et al., 1995) \\
\hline 17 & ARI KOUKOURI & 13.9167 & 13.1000 & 270 & Niger & $5488-9617$ & (Schulz et al., 1995) \\
\hline 18 & ARI KOUKOURI 85 & 13.9167 & 13.1000 & 270 & Niger & 7250 & (Schulz et al., 1995) \\
\hline 19 & ENNERI ACHELOUMA [Profil XII] & 22.3500 & 12.7000 & 650 & Niger & 7806 & (Schulz, 1980) \\
\hline 20 & SETTAFET & 25.3500 & 11.4333 & 1100 & $\begin{array}{r}\text { Libyan } \\
\text { Arab } \\
\text { Jamahiriya }\end{array}$ & 4710 & (Schulz, 1980) \\
\hline
\end{tabular}




\begin{tabular}{|c|c|c|c|c|c|c|c|}
\hline 21 & UAN AFUDA CAVE & 24.8678 & 10.5003 & 922 & $\begin{array}{r}\text { Libyan } \\
\text { Arab } \\
\text { Jamahiriya } \\
\end{array}$ & $8836-9835$ & (Mercuri, 1999) \\
\hline 22 & UAN MUHUGGIAG & 24.8387 & 10.5078 & 915 & $\begin{array}{r}\text { Libyan } \\
\text { Arab } \\
\text { Jamahiriya }\end{array}$ & $4496-7758$ & (Mercuri et al., 1998) \\
\hline 23 & UAN TABU & 24.8558 & 10.5237 & 915 & $\begin{array}{r}\text { Libyan } \\
\text { Arab } \\
\text { Jamahiriya } \\
\end{array}$ & 4218 - 9933 & $\begin{array}{r}\text { (Mercuri and Trevisan } \\
\text { Grandi, 2001) }\end{array}$ \\
\hline 24 & SHATI & 27.5000 & 13.8333 & 305 & $\begin{array}{r}\text { Libyan } \\
\text { Arab } \\
\text { Jamahiriya } \\
\end{array}$ & 6350 & (Delibrias et al., 1982) \\
\hline 25 & BAL & 13.3042 & 10.9430 & 300 & Nigeria & $341-11905$ & $\begin{array}{l}\text { (Salzmann and Waller, } \\
\text { 1998; Salzmann, 1999) }\end{array}$ \\
\hline 26 & KAIGAMA & 13.2510 & 11.5675 & 330 & Nigeria & $3567-11112$ & $\begin{array}{r}\text { (Salzmann, 1996; } \\
\text { Salzmann and Waller, } \\
\text { 1998) }\end{array}$ \\
\hline 27 & KAJEMARUM & 13.3030 & 11.0240 & 300 & Nigeria & $2845-11027$ & $\begin{array}{r}\text { (Salzmann and Waller, } \\
\text { 1998) }\end{array}$ \\
\hline 28 & KULUWU & 13.2170 & 11.5505 & 330 & Nigeria & $4703-11406$ & $\begin{array}{r}\text { (Salzmann and Waller, } \\
1998)\end{array}$ \\
\hline 29 & TILLA & 10.3907 & 12.1245 & 690 & Nigeria & $248-13064$ & $\begin{array}{r}\text { (Salzmann, 2000; } \\
\text { Salzmann et al., 2002) }\end{array}$ \\
\hline 30 & DIOGO 1 & 15.2667 & -16.8000 & 8 & Senegal & 9990 & (Lézine, 1987) \\
\hline 31 & DIOGO 2 & 15.2667 & -16.8000 & 8 & Senegal & $450-11412$ & (Lézine, 1988a) \\
\hline 32 & GUIERS 02 & 16.1167 & -15.9167 & 0 & Senegal & $162-6703$ & (Lézine, 1988b) \\
\hline 33 & GUIERS 03 & 16.2833 & -15.8333 & 1 & Senegal & $1121-6764$ & (Lézine, 1988b) \\
\hline 34 & LOMPOUL & 15.4167 & -16.7167 & 3 & Senegal & 2008 - 10309 & $\begin{array}{r}\text { (Lézine, 1987, 1988a; } \\
\text { Lézine and Chateauneuf, } \\
\text { 1991) }\end{array}$ \\
\hline
\end{tabular}




\begin{tabular}{|c|c|c|c|c|c|c|c|}
\hline 35 & POTOU & 15.1667 & -16.8667 & 11 & Senegal & $193-11399$ & $\begin{array}{r}\text { (Lézine, 1987, 1988a; } \\
\text { Lézine and Chateauneuf, }\end{array}$ \\
\hline 36 & TIGUENT & 17.25 & -16.0166 & $\mathrm{~N} / \mathrm{A}$ & Mauritania & 3342 & (Medus and Barbey, 1979) \\
\hline 37 & KISSI (BS6) & 14.6183 & -0.1367 & 280 & $\begin{array}{r}\text { Burkina } \\
\text { Faso } \\
\end{array}$ & 567 & (Ballouche, 1998) \\
\hline 38 & OURSI & 14.6528 & -0.4860 & 290 & $\begin{array}{r}\text { Burkina } \\
\text { Faso } \\
\end{array}$ & $52-3265$ & $\begin{array}{r}\text { (Ballouche and Neumann, } \\
\text { 1995) } \\
\end{array}$ \\
\hline 39 & BIR ATRUN & 18.1667 & 26.6500 & 600 & Sudan & $6469-10229$ & $\begin{array}{r}\text { (Ritchie, 1987; Ritchie and } \\
\text { Haynes, 1987) }\end{array}$ \\
\hline 40 & EL ATRUN & 18.0167 & 27.1500 & 600 & Sudan & 10334 & (Jahns, 1995) \\
\hline 41 & OYO & 19.2560 & 26.1747 & 510 & Sudan & $5187-10363$ & $\begin{array}{r}\text { (Ritchie et al., 1985; } \\
\text { Ritchie, 1994) }\end{array}$ \\
\hline 42 & SELIMA & 21.3667 & 29.3060 & 270 & Sudan & $7643-11021$ & $\begin{array}{r}\text { (Ritchie and Haynes, 1987; } \\
\text { Haynes et al., 1989) }\end{array}$ \\
\hline 43 & THIAYE & 14.9167 & -17.5000 & 1 & Senegal & $5593-6371$ & $\begin{array}{r}\text { (Lézine et al., 1985; Lézine, } \\
\text { 1987) }\end{array}$ \\
\hline 44 & $\begin{array}{lll}\begin{array}{l}\text { GABRONG [ENNERI DIRENNAO] } \\
\text { (GABR) }\end{array} & \\
\end{array}$ & 21.5000 & 17.1167 & 1115 & Chad & 8945 & (Schulz, 1980) \\
\hline 45 & TARSO YEGA GAVRILOVIC (GAVR 1) & 20.6667 & 17.5000 & 2000 & Chad & 8930 & (Schulz, 1980) \\
\hline 46 & ENNERI BARDAGUE [JAMT36] & 21.4167 & 16.9167 & $\mathrm{~N} / \mathrm{A}$ & Chad & 9294 & (Schulz, 1980) \\
\hline 47 & YEBBI [GRA] & 20.8667 & 18.0383 & 1440 & Chad & 9136 & (Schulz, 1980) \\
\hline 48 & YOA & 19.05 & 20.5166 & 380 & Chad & $0-6034$ & $\begin{array}{r}\text { (Kröpelin et al., 2008; } \\
\text { Lézine, 2009) }\end{array}$ \\
\hline
\end{tabular}


Table S2. Classification of pollen taxa into Saharan Sahelian, Sudanian, and/or GuineoCongolian groups according to the phytogeographical affinities of the species they represent. A pollen taxon is considered as exclusive to a given group when it presents phytogeographical affinities with only this group. The taxa nomenclature is from the African Pollen Database (Vincens et al., 2007) and respects the original determination provided by authors.

\begin{tabular}{|c|c|c|c|c|}
\hline Taxon Name [APD] & Guineo-Congolian & Sudanian & Sahelian & Saharan \\
\hline Acanthus-type & $x$ & & & \\
\hline Afraegle-type paniculata & $\mathrm{x}$ & & & \\
\hline Ammannia-type & $\mathrm{x}$ & & & \\
\hline Anthocleista & $\mathrm{x}$ & & & \\
\hline Anthostema-type & $\mathrm{x}$ & & & \\
\hline Anthostema-type senegalense & $\mathrm{x}$ & & & \\
\hline Brachystegia & $\mathrm{x}$ & & & \\
\hline Caesalpinia-type & $x$ & & & \\
\hline Chrysobalanus icaco/Parinari & $x$ & & & \\
\hline Connaraceae undiff. & $x$ & & & \\
\hline Dodonaea & $\mathrm{x}$ & & & \\
\hline Dodonaea viscosa-type & $\mathrm{x}$ & & & \\
\hline Dombeya-type buettneri & $\mathrm{x}$ & & & \\
\hline Dombeya-type quinqueseta & $\mathrm{x}$ & & & \\
\hline Elaeis guineensis & $\mathrm{x}$ & & & \\
\hline Eremospatha & $\mathrm{x}$ & & & \\
\hline Erythrococca-type africana & $\mathrm{x}$ & & & \\
\hline Garcinia & $\mathrm{x}$ & & & \\
\hline Harungana & $\mathrm{x}$ & & & \\
\hline Harungana madagascariensis-type & $x$ & & & \\
\hline Hewittia-type scandens & $\mathrm{x}$ & & & \\
\hline Landolphia-type & $\mathrm{x}$ & & & \\
\hline Lophira alata-type & $x$ & & & \\
\hline Mallotus-type & $x$ & & & \\
\hline Meliaceae undiff. & $\mathrm{x}$ & & & \\
\hline Millettia-type & $\mathrm{x}$ & & & \\
\hline Myrianthus-type & $x$ & & & \\
\hline Myrianthus-type serratus & $x$ & & & \\
\hline Naucleae undiff. & $\mathrm{x}$ & & & \\
\hline Nauclea-type & $x$ & & & \\
\hline Pancovia-type & $\mathrm{x}$ & & & \\
\hline Pancovia-type bijuga & $\mathrm{x}$ & & & \\
\hline Sorindeia-type juglandifolia & $\mathrm{x}$ & & & \\
\hline Thunbergia & $x$ & & & \\
\hline
\end{tabular}




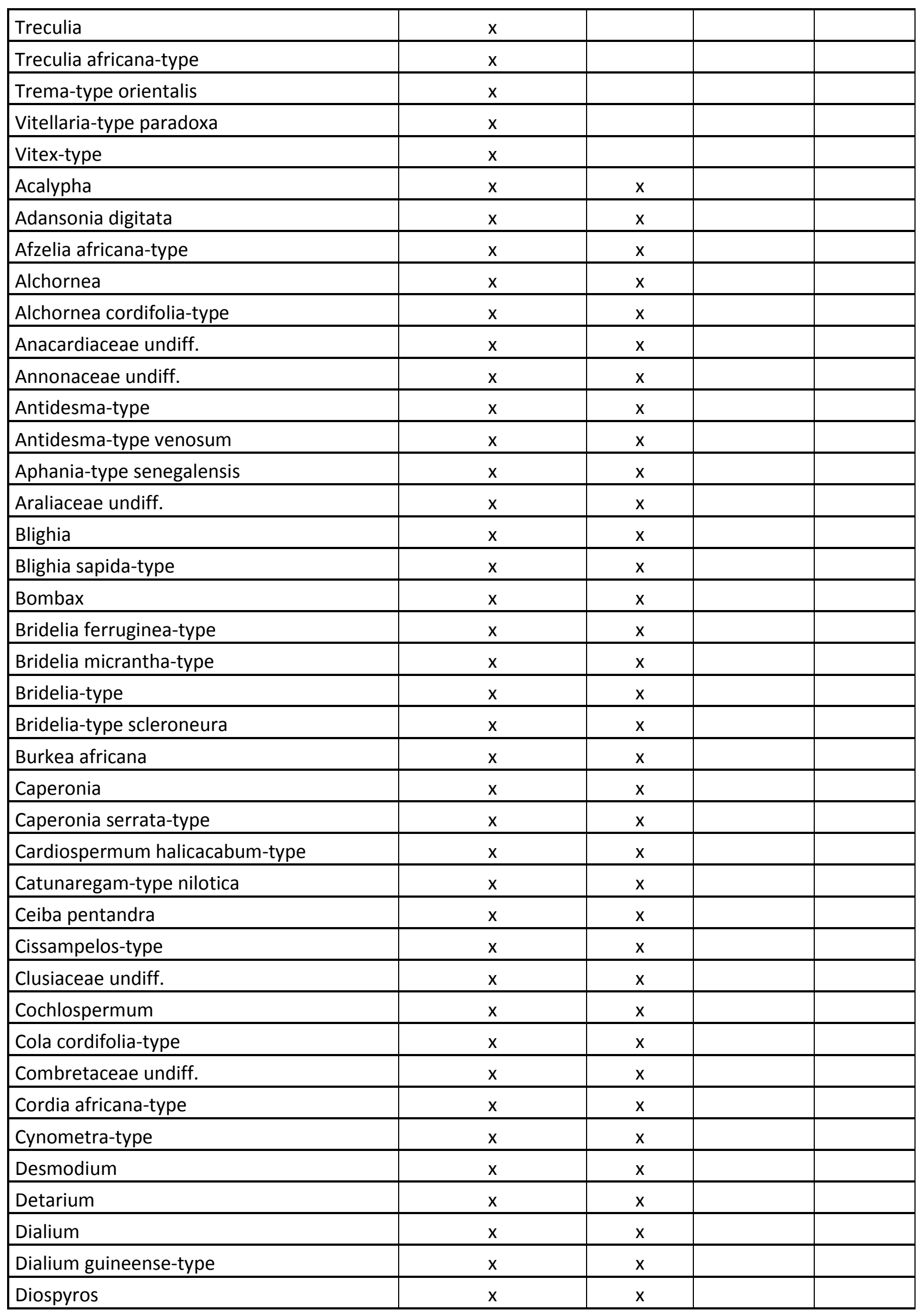




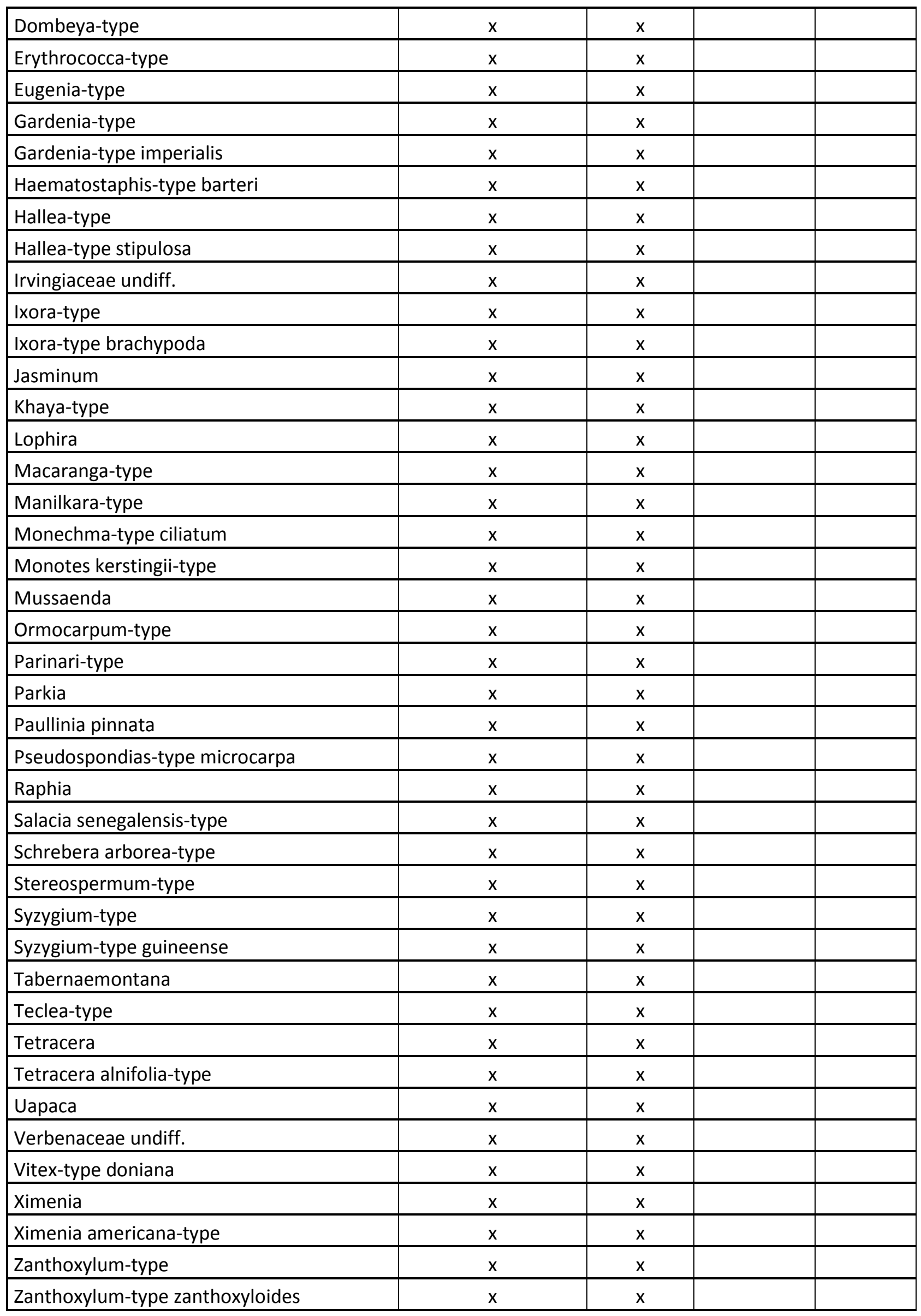




\begin{tabular}{|c|c|c|c|c|}
\hline Albizia-type & $x$ & $x$ & $x$ & \\
\hline Allophylus & $x$ & $x$ & $x$ & \\
\hline Allophylus africanus-type & $x$ & $x$ & $x$ & \\
\hline Celtis & $x$ & $x$ & $x$ & \\
\hline Combretum-type & $x$ & $x$ & $x$ & \\
\hline Dalechampia & $\mathrm{x}$ & $\mathrm{x}$ & $x$ & \\
\hline Dichrostachys & $x$ & $x$ & $x$ & \\
\hline Gnidia-type & $x$ & $x$ & $x$ & \\
\hline Hemizygia bracteosa-type & $\mathrm{x}$ & $x$ & $x$ & \\
\hline Hymenocardia & $\mathrm{x}$ & $\mathrm{x}$ & $x$ & \\
\hline Hymenocardia acida-type & $\mathrm{x}$ & $\mathrm{x}$ & $x$ & \\
\hline Mitragyna-type inermis & $\mathrm{x}$ & $\mathrm{x}$ & $x$ & \\
\hline Morelia senegalensis & $\mathrm{x}$ & $\mathrm{x}$ & $\mathrm{x}$ & \\
\hline Musanga-type & $\mathrm{x}$ & $\mathrm{x}$ & $\mathrm{x}$ & \\
\hline Piliostigma reticulatum-type & $\mathrm{x}$ & $\mathrm{x}$ & $\mathrm{x}$ & \\
\hline Pseudocedrela kotschyi & $x$ & $\mathrm{x}$ & $\mathrm{x}$ & \\
\hline Pterocarpus-type & $x$ & $x$ & $\mathrm{x}$ & \\
\hline Sapindaceae undiff. & $x$ & $\mathrm{x}$ & $\mathrm{x}$ & \\
\hline Tapinanthus-type & $x$ & $\mathrm{x}$ & $\mathrm{x}$ & \\
\hline Acanthaceae undiff. & $x$ & $\mathrm{x}$ & $x$ & $\mathrm{x}$ \\
\hline Apocynaceae undiff. & $x$ & $\mathrm{x}$ & $x$ & $\mathrm{x}$ \\
\hline Asteraceae undiff. & $x$ & $x$ & $x$ & $x$ \\
\hline Caesalpiniaceae undiff. & $x$ & $\mathrm{x}$ & $\mathrm{x}$ & $\mathrm{x}$ \\
\hline Campanulaceae undiff. & $\mathrm{x}$ & $\mathrm{x}$ & $\mathrm{x}$ & $x$ \\
\hline Euphorbiaceae undiff. & $\mathrm{x}$ & $\mathrm{x}$ & $x$ & $x$ \\
\hline Farsetia & $\mathrm{x}$ & $\mathrm{x}$ & $\mathrm{x}$ & $x$ \\
\hline Lippia-type & $\mathrm{x}$ & $\mathrm{x}$ & $\mathrm{x}$ & $\mathrm{x}$ \\
\hline Mimosaceae undiff. & $x$ & $\mathrm{x}$ & $x$ & $x$ \\
\hline Moraceae undiff. & $x$ & $\mathrm{x}$ & $x$ & $\mathrm{x}$ \\
\hline Myrtaceae undiff. & $x$ & $\mathrm{x}$ & $x$ & $x$ \\
\hline Phoenix & $\mathrm{x}$ & $x$ & $x$ & $x$ \\
\hline Phoenix reclinata-type & $\mathrm{x}$ & $\mathrm{x}$ & $x$ & $x$ \\
\hline Rubiaceae undiff. & $x$ & $\mathrm{x}$ & $x$ & $x$ \\
\hline Rutaceae undiff. & $x$ & $x$ & $x$ & $\mathrm{x}$ \\
\hline Sapotaceae undiff. & $\mathrm{x}$ & $\mathrm{x}$ & $x$ & $x$ \\
\hline Acalypha ciliata-type & & $\mathrm{x}$ & & \\
\hline Acalypha crenata-type & & $x$ & & \\
\hline Adansonia & & $x$ & & \\
\hline Alysicarpus & & $x$ & & \\
\hline Basilicum-type polystachyon & & $\mathrm{x}$ & & \\
\hline Borassus/Hyphaene & & $x$ & & \\
\hline
\end{tabular}




\begin{tabular}{|c|c|c|c|}
\hline Capparis tomentosa-type & $x$ & & \\
\hline Cayratia-type & $x$ & & \\
\hline Cissus & $x$ & & \\
\hline Clerodendrum & $\mathrm{x}$ & & \\
\hline Combretum-type molle & $x$ & & \\
\hline Crossopteryx febrifuga & $\mathrm{x}$ & & \\
\hline Cussonia & $\mathrm{x}$ & & \\
\hline Entada/Prosopis & $\mathrm{x}$ & & \\
\hline Entada-type & $\mathrm{x}$ & & \\
\hline Eriosema-type & $\mathrm{x}$ & & \\
\hline Flueggea virosa-type & $\mathrm{x}$ & & \\
\hline Hygrophila & $\mathrm{x}$ & & \\
\hline Isoberlinia-type & $\mathrm{x}$ & & \\
\hline Kedrostis & $\mathrm{x}$ & & \\
\hline Lannea/Sclerocarya & $\mathrm{x}$ & & \\
\hline Maytenus & $x$ & & \\
\hline Micrococca-type mercurialis & $x$ & & \\
\hline Oxalis & $\mathrm{x}$ & & \\
\hline Pavetta & $\mathrm{x}$ & & \\
\hline Pentodon-type pentandrus & $\mathrm{x}$ & & \\
\hline Rhus-type longipes & $\mathrm{x}$ & & \\
\hline Salacia & $\mathrm{x}$ & & \\
\hline Sida & $x$ & & \\
\hline Sopubia-type parviflora & $\mathrm{x}$ & & \\
\hline Spondias monbin-type & $\mathrm{x}$ & & \\
\hline Sterculia-type & $\mathrm{x}$ & & \\
\hline Sterculia-type setigera & $\mathrm{x}$ & & \\
\hline Strychnos & $\mathrm{x}$ & & \\
\hline Tacazzea-type apiculata & $\mathrm{x}$ & & \\
\hline Tacca leontopetaloides & $\mathrm{x}$ & & \\
\hline Vernonia perrottetii-type & $\mathrm{x}$ & & \\
\hline Vernonia-type galamensis & $\mathrm{x}$ & & \\
\hline Vigna-type & $\mathrm{x}$ & & \\
\hline Zornia & $x$ & & \\
\hline Acacia ehrenbergiana-type & $\mathrm{x}$ & $x$ & \\
\hline Acacia gummifera-type & $x$ & $x$ & \\
\hline Acacia nilotica-type & $\mathrm{x}$ & $x$ & \\
\hline Acacia polyacantha-type & $\mathrm{x}$ & $x$ & \\
\hline Acacia senegal-type & $\mathrm{x}$ & $x$ & \\
\hline Acacia tortilis-type & $\mathrm{x}$ & $x$ & \\
\hline Adenium obesum-type & $x$ & $x$ & \\
\hline
\end{tabular}




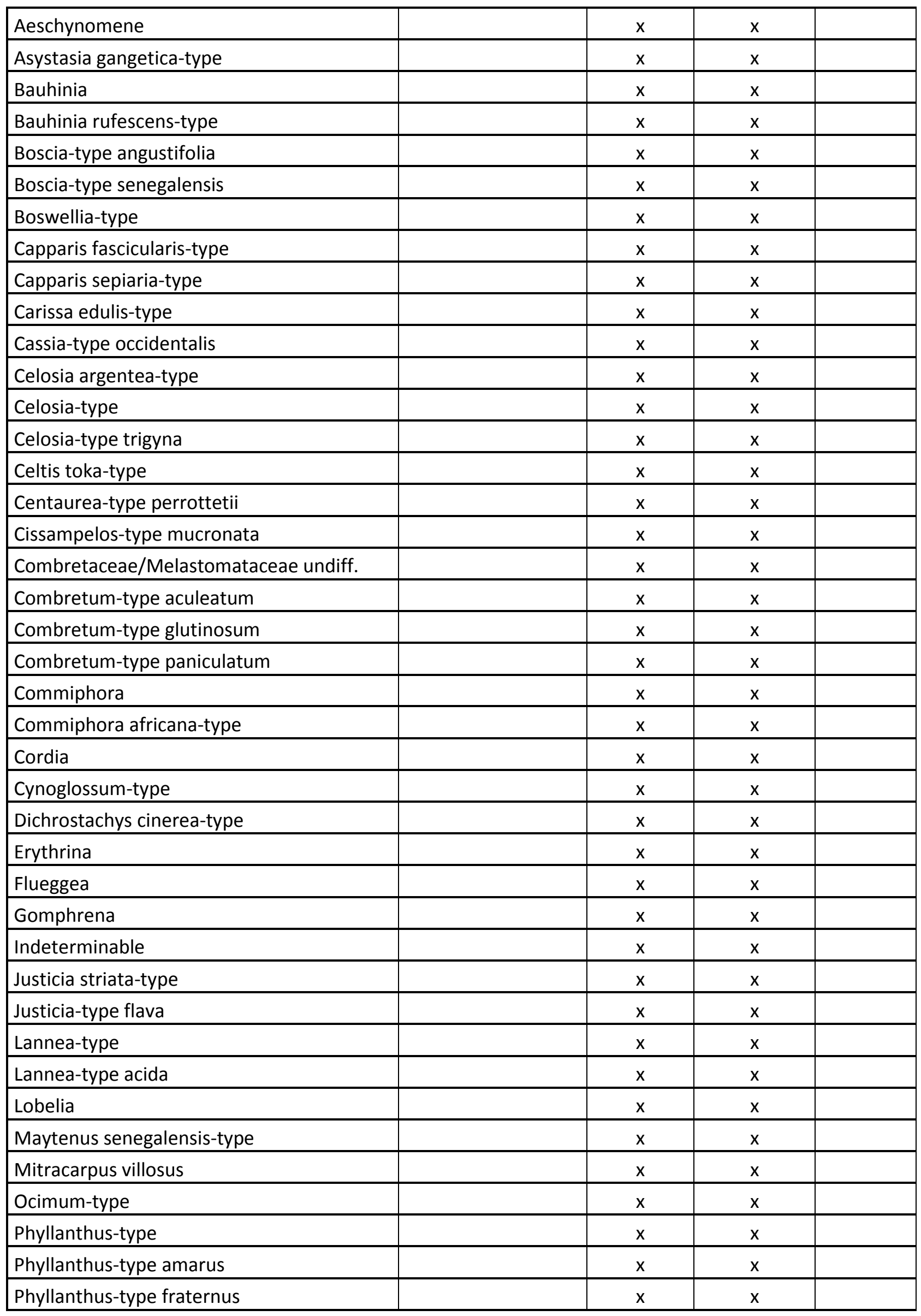




\begin{tabular}{|c|c|c|c|}
\hline Phyllanthus-type maderaspatensis & $\mathrm{x}$ & $x$ & \\
\hline Phyllanthus-type reticulatus & $x$ & $x$ & \\
\hline Piliostigma & $\mathrm{x}$ & $x$ & \\
\hline Pluchea & $\mathrm{x}$ & $x$ & \\
\hline Prosopis-type africana & $\mathrm{x}$ & $x$ & \\
\hline Spermacoce-type & $\mathrm{x}$ & $\mathrm{x}$ & \\
\hline Spermacoce-type chaetocephala & $\mathrm{x}$ & $x$ & \\
\hline Spermacoce-type radiata & $\mathrm{x}$ & $x$ & \\
\hline Triumfetta-type & $\mathrm{x}$ & $\mathrm{x}$ & \\
\hline Waltheria & $\mathrm{x}$ & $x$ & \\
\hline Acacia & $\mathrm{x}$ & $\mathrm{x}$ & $x$ \\
\hline Acacia seyal-type & $\mathrm{x}$ & $\mathrm{x}$ & $x$ \\
\hline Achyranthes-type aspera & $\mathrm{x}$ & $\mathrm{x}$ & $x$ \\
\hline Amaranthaceae undiff. & $\mathrm{x}$ & $x$ & $\underline{x}$ \\
\hline Amaranthaceae/Chenopodiaceae undiff. & $\mathrm{x}$ & $\mathrm{x}$ & $\underline{x}$ \\
\hline Apiaceae undiff. & $x$ & $x$ & $x$ \\
\hline Asclepiadaceae undiff. & $x$ & $x$ & $x$ \\
\hline Asparagus & $\mathrm{x}$ & $x$ & $x$ \\
\hline Balanites & $x$ & $x$ & $x$ \\
\hline Balanites aegyptiaca-type & $x$ & $\mathrm{x}$ & $x$ \\
\hline Barleria & $x$ & $x$ & $x$ \\
\hline Blepharis-type & $x$ & $x$ & $x$ \\
\hline Boerhavia-type & $x$ & $x$ & $x$ \\
\hline Boscia-type & $x$ & $x$ & $x$ \\
\hline Boscia-type salicifolia & $x$ & $x$ & $x$ \\
\hline Cadaba-type & $\mathrm{x}$ & $\mathrm{x}$ & $x$ \\
\hline Cadaba-type farinosa & $\mathrm{x}$ & $\mathrm{x}$ & $\underline{x}$ \\
\hline Cadaba-type glandulosa & $x$ & $x$ & $x$ \\
\hline Capparidaceae undiff. & $x$ & $x$ & $x$ \\
\hline Capparis & $\mathrm{x}$ & $x$ & $x$ \\
\hline Capparis decidua-type & $\mathrm{x}$ & $x$ & $x$ \\
\hline Caryophyllaceae undiff. & $\mathrm{x}$ & $x$ & $x$ \\
\hline Cassia-type & $\mathrm{x}$ & $x$ & $x$ \\
\hline Celastraceae undiff. & $x$ & $x$ & $x$ \\
\hline Chenopodiaceae undiff. & $\mathrm{x}$ & $x$ & $x$ \\
\hline Chenopodium-type & $x$ & $x$ & $x$ \\
\hline Chrozophora & $\mathrm{x}$ & $x$ & $x$ \\
\hline Citrullus & $\mathrm{x}$ & $x$ & $x$ \\
\hline Clematis-type & $\mathrm{x}$ & $x$ & $x$ \\
\hline Clematis-type hirsuta & $\mathrm{x}$ & $x$ & $x$ \\
\hline Cleome brachycarpa-type & $x$ & $x$ & $x$ \\
\hline
\end{tabular}




\begin{tabular}{|c|c|c|c|}
\hline Cleome-type & $x$ & $x$ & $\mathrm{x}$ \\
\hline Cleome-type gynandra & $x$ & $x$ & $\mathrm{x}$ \\
\hline Clutia & $x$ & $x$ & $\mathrm{x}$ \\
\hline Coccinia & $x$ & $x$ & $x$ \\
\hline Cocculus & $x$ & $x$ & $x$ \\
\hline Cocculus pendulus-type & $\mathrm{x}$ & $x$ & $\mathrm{x}$ \\
\hline Commelinaceae undiff. & $x$ & $x$ & $\mathrm{x}$ \\
\hline Commelina-type & $x$ & $x$ & $\mathrm{x}$ \\
\hline Commelina-type benghalensis & $x$ & $x$ & $\mathrm{x}$ \\
\hline Convolvulaceae undiff. & $\mathrm{x}$ & $\mathrm{x}$ & $x$ \\
\hline Convolvulus-type & $\mathrm{x}$ & $\mathrm{x}$ & $x$ \\
\hline Corchorus-type & $\mathrm{x}$ & $\mathrm{x}$ & $x$ \\
\hline Cotula-type anthemoides & $\mathrm{x}$ & $\mathrm{x}$ & $x$ \\
\hline Crassula & $\mathrm{x}$ & $\mathrm{x}$ & $\underline{x}$ \\
\hline Crateva adansonii & $\mathrm{x}$ & $\mathrm{x}$ & $\underline{x}$ \\
\hline Crotalaria & $\mathrm{x}$ & $\mathrm{x}$ & $x$ \\
\hline Croton/Jatropha & $\mathrm{x}$ & $\mathrm{x}$ & $x$ \\
\hline Croton-type & $\mathrm{x}$ & $\mathrm{x}$ & $x$ \\
\hline Cucumis & $\mathrm{x}$ & $\mathrm{x}$ & $x$ \\
\hline Cuscuta-type & $\mathrm{x}$ & $x$ & $\mathrm{x}$ \\
\hline Euphorbia-type & $\mathrm{x}$ & $x$ & $\mathrm{x}$ \\
\hline Faidherbia albida & $x$ & $x$ & $\mathrm{x}$ \\
\hline Ficus & $\mathrm{x}$ & $x$ & $x$ \\
\hline Gentianaceae undiff. & $\mathrm{x}$ & $\mathrm{x}$ & $x$ \\
\hline Geraniaceae undiff. & $\mathrm{x}$ & $x$ & $x$ \\
\hline Grewia-type & $\mathrm{x}$ & $\mathrm{x}$ & $\underline{x}$ \\
\hline Heliotropium & $\mathrm{x}$ & $\mathrm{x}$ & $\underline{x}$ \\
\hline Heliotropium strigosum-type & $\mathrm{x}$ & $x$ & 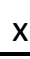 \\
\hline Hibiscus & $\mathrm{x}$ & $x$ & $x$ \\
\hline Hypericum & $\mathrm{x}$ & $x$ & $\mathrm{x}$ \\
\hline Hyphaene-type & $\mathrm{x}$ & $x$ & $x$ \\
\hline Indigofera & $\mathrm{x}$ & $x$ & $x$ \\
\hline Kohautia & $\mathrm{x}$ & $\mathrm{x}$ & $x$ \\
\hline Lamiaceae undiff. & $\mathrm{x}$ & $\mathrm{x}$ & $x$ \\
\hline Lotus arabicus-type & $\mathrm{x}$ & $x$ & $x$ \\
\hline Maerua/Ritchiea & $\mathrm{x}$ & $x$ & $x$ \\
\hline Maerua-type & $\mathrm{x}$ & $x$ & $\mathrm{x}$ \\
\hline Maerua-type crassifolia & $\mathrm{x}$ & $\mathrm{x}$ & $x$ \\
\hline Malvaceae undiff. & $x$ & $\mathrm{x}$ & $x$ \\
\hline Menispermaceae undiff. & $\mathrm{x}$ & $x$ & $x$ \\
\hline Mentha-type & $x$ & $x$ & $\mathrm{x}$ \\
\hline
\end{tabular}




\begin{tabular}{|c|c|c|c|}
\hline Merremia-type & $\mathrm{x}$ & $x$ & $x$ \\
\hline Monsonia & $x$ & $x$ & $\mathrm{x}$ \\
\hline Nyctaginaceae undiff. & $\mathrm{x}$ & $\mathrm{x}$ & $\mathrm{x}$ \\
\hline Oldenlandia-type & $\mathrm{x}$ & $\mathrm{x}$ & $\mathrm{x}$ \\
\hline Palmae undiff. & $\mathrm{x}$ & $\mathrm{x}$ & $\mathrm{x}$ \\
\hline Pavonia & $\mathrm{x}$ & $\mathrm{x}$ & $\mathrm{x}$ \\
\hline Peristrophe-type & $\mathrm{x}$ & $\mathrm{x}$ & $\mathrm{x}$ \\
\hline Poaceae undiff. & $\mathrm{x}$ & $\mathrm{x}$ & $\mathrm{x}$ \\
\hline Polycarpaea-type & $\mathrm{x}$ & $\mathrm{x}$ & $x$ \\
\hline Polycarpon-type & $\mathrm{x}$ & $\mathrm{x}$ & $x$ \\
\hline Polycarpon-type prostratum & $\mathrm{x}$ & $\mathrm{x}$ & $x$ \\
\hline Ranunculaceae undiff. & $\mathrm{x}$ & $x$ & $\mathrm{x}$ \\
\hline Rhamnaceae undiff. & $\mathrm{x}$ & $x$ & $\mathrm{x}$ \\
\hline Rhus-type & $x$ & $x$ & $\mathrm{x}$ \\
\hline Rhynchosia-type & $\mathrm{x}$ & $\mathrm{x}$ & $\mathrm{x}$ \\
\hline Ricinus communis & $\mathrm{x}$ & $\mathrm{x}$ & $\mathrm{x}$ \\
\hline Ruellia & $\mathrm{x}$ & $\mathrm{x}$ & $\mathrm{x}$ \\
\hline Rumex & $\mathrm{x}$ & $\mathrm{x}$ & $\mathrm{x}$ \\
\hline Salix & $\mathrm{x}$ & $\mathrm{x}$ & $\mathrm{x}$ \\
\hline Salvadora & $\mathrm{x}$ & $\mathrm{x}$ & $\mathrm{x}$ \\
\hline Salvadora persica-type & $\mathrm{x}$ & $\mathrm{x}$ & $\mathrm{x}$ \\
\hline Salvadoraceae undiff. & $\mathrm{x}$ & $\mathrm{x}$ & $x$ \\
\hline Sesbania & $\mathrm{x}$ & $\mathrm{x}$ & $\mathrm{x}$ \\
\hline Solanaceae undiff. & $\mathrm{x}$ & $\mathrm{x}$ & $x$ \\
\hline Solanum-type & $\mathrm{x}$ & $x$ & $\mathrm{x}$ \\
\hline Tamarindus-type indica & $\mathrm{x}$ & $x$ & $\mathrm{x}$ \\
\hline Tephrosia & $\mathrm{x}$ & $x$ & $\mathrm{x}$ \\
\hline Thymelaeaceae undiff. & $\mathrm{x}$ & $x$ & $\mathrm{x}$ \\
\hline Tiliaceae undiff. & $\mathrm{x}$ & $\mathrm{x}$ & $x$ \\
\hline Tinospora bakis-type & $\mathrm{x}$ & $\mathrm{x}$ & $x$ \\
\hline Trichodesma & $x$ & $\mathrm{x}$ & $\mathrm{x}$ \\
\hline Trichodesma africanum-type & $\mathrm{x}$ & $\mathrm{x}$ & $x$ \\
\hline Vahlia & $\mathrm{x}$ & $\mathrm{x}$ & $\mathrm{x}$ \\
\hline Zygophyllaceae undiff. & $\mathrm{x}$ & $\mathrm{x}$ & $\underline{x}$ \\
\hline Cayratia-type ibuensis & & $\mathrm{x}$ & \\
\hline Guiera senegalensis & & $\mathrm{x}$ & \\
\hline Lamiaceae type 6 (exine type Leucas) & & $\mathrm{x}$ & \\
\hline Saxifraga hederifolia & & $\mathrm{x}$ & \\
\hline Abutilon & & $\mathrm{x}$ & $\underline{x}$ \\
\hline Aerva-type javanica & & $x$ & $x$ \\
\hline Aerva-type lanata & & $x$ & $\mathrm{x}$ \\
\hline
\end{tabular}




\begin{tabular}{|c|c|c|}
\hline Aizoaceae undiff. & $x$ & $x$ \\
\hline Alternanthera & $x$ & $x$ \\
\hline Ambrosia-type maritima & $x$ & $\mathrm{x}$ \\
\hline Arnebia hispidissima-type & $x$ & $x$ \\
\hline Brassicaceae undiff. & $x$ & $x$ \\
\hline Cassia-type italica & $x$ & $\mathrm{x}$ \\
\hline Centaurea-type senegalensis & $x$ & $\mathrm{x}$ \\
\hline Chascanum & $x$ & $\mathrm{x}$ \\
\hline Cichorieae undiff. & $x$ & $\mathrm{x}$ \\
\hline Cirsium & $x$ & $\mathrm{x}$ \\
\hline Cleome-type arabica & $x$ & $\mathrm{x}$ \\
\hline Cleome-type monophylla & $\mathrm{x}$ & $\mathrm{x}$ \\
\hline Cleome-type scaposa & $\mathrm{x}$ & $\mathrm{x}$ \\
\hline Commicarpus-type & $\mathrm{x}$ & $\mathrm{x}$ \\
\hline Cynareae undiff. & $\mathrm{x}$ & $\mathrm{x}$ \\
\hline Euphorbia-type hirta & $\mathrm{x}$ & $x$ \\
\hline Galium-type & $x$ & $\mathrm{x}$ \\
\hline Helichrysum-type & $x$ & $\mathrm{x}$ \\
\hline Hyphaene-type thebaïca & $\mathrm{x}$ & $x$ \\
\hline Ipomoea-type & $x$ & $\mathrm{x}$ \\
\hline Lactuceae undiff. & $x$ & $\mathrm{x}$ \\
\hline Lathyrus/Vicia & $x$ & $x$ \\
\hline Leucas-type & $x$ & $x$ \\
\hline Limeum & $x$ & $\mathrm{x}$ \\
\hline Luffa-type & $x$ & $\mathrm{x}$ \\
\hline Lythraceae undiff. & $\mathrm{x}$ & $\underline{x}$ \\
\hline Nucularia perrini & $\mathrm{x}$ & $\underline{x}$ \\
\hline Phyla-type nodiflora & $\mathrm{x}$ & $x$ \\
\hline Plantago lanceolata-type & $x$ & $x$ \\
\hline Polygala-type & $x$ & $\mathrm{x}$ \\
\hline Psilotrichum-type & $x$ & $\mathrm{x}$ \\
\hline Psoralea & $x$ & $\mathrm{x}$ \\
\hline Pulicaria & $x$ & $x$ \\
\hline Resedaceae undiff. & $\mathrm{x}$ & $\mathrm{x}$ \\
\hline Suaeda & $x$ & $\mathrm{x}$ \\
\hline Tamarix & $x$ & $x$ \\
\hline Tamarix senegalensis-type & $x$ & $\mathrm{x}$ \\
\hline Tribulus & $x$ & $x$ \\
\hline Tribulus terrestris-type & $x$ & $x$ \\
\hline Veronica & $\mathrm{x}$ & $x$ \\
\hline Zygophyllum & $x$ & $\mathrm{x}$ \\
\hline
\end{tabular}




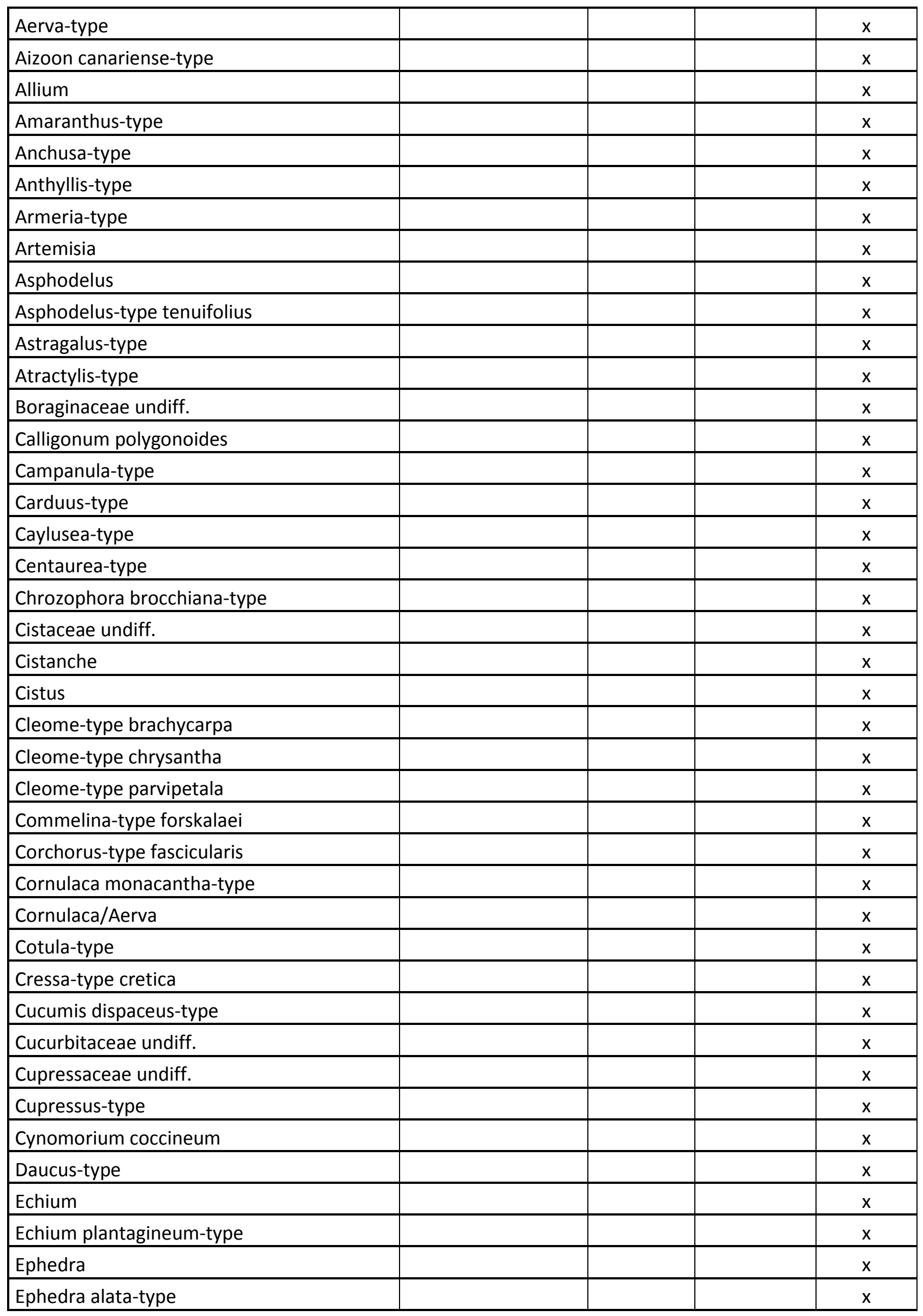




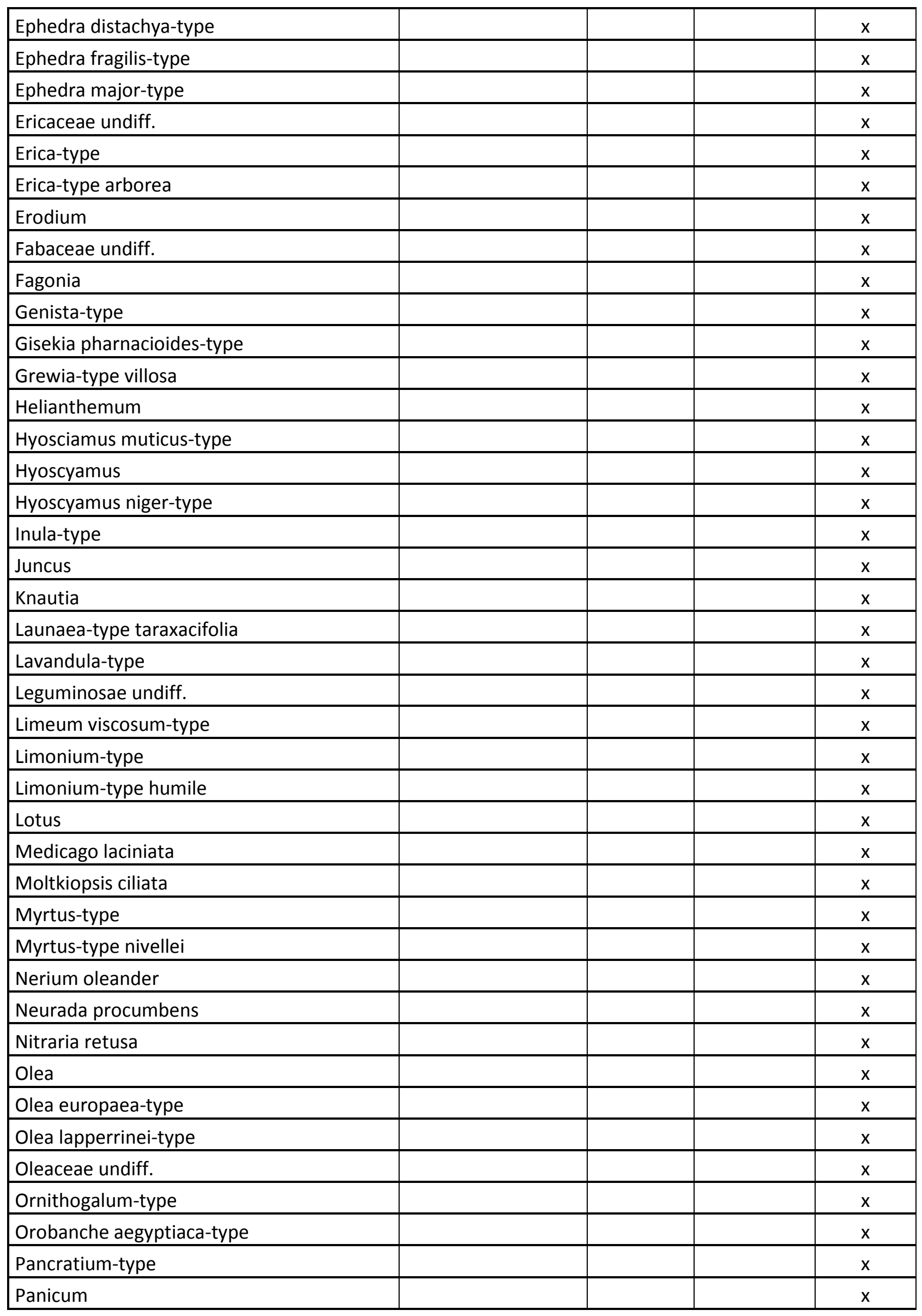




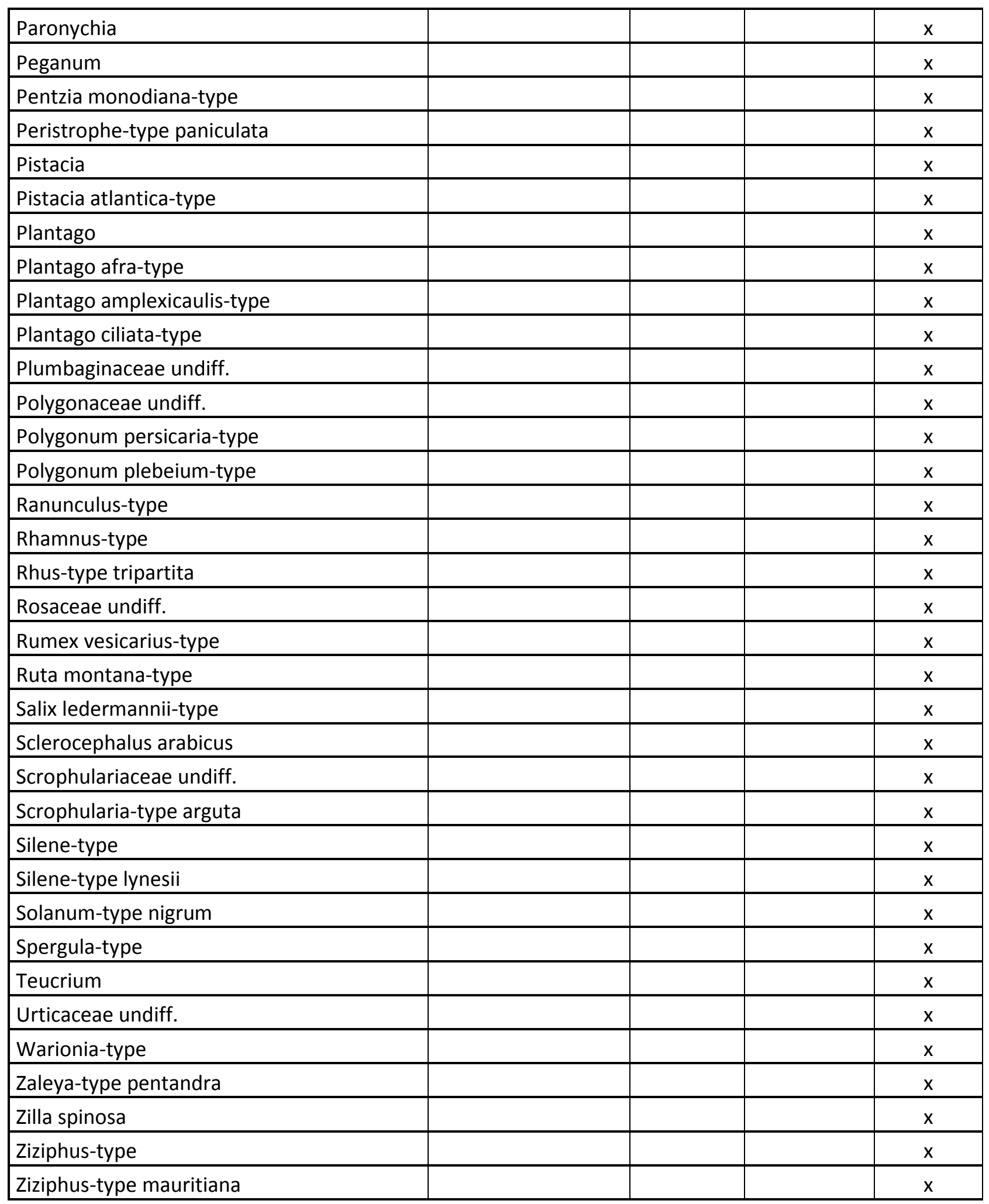



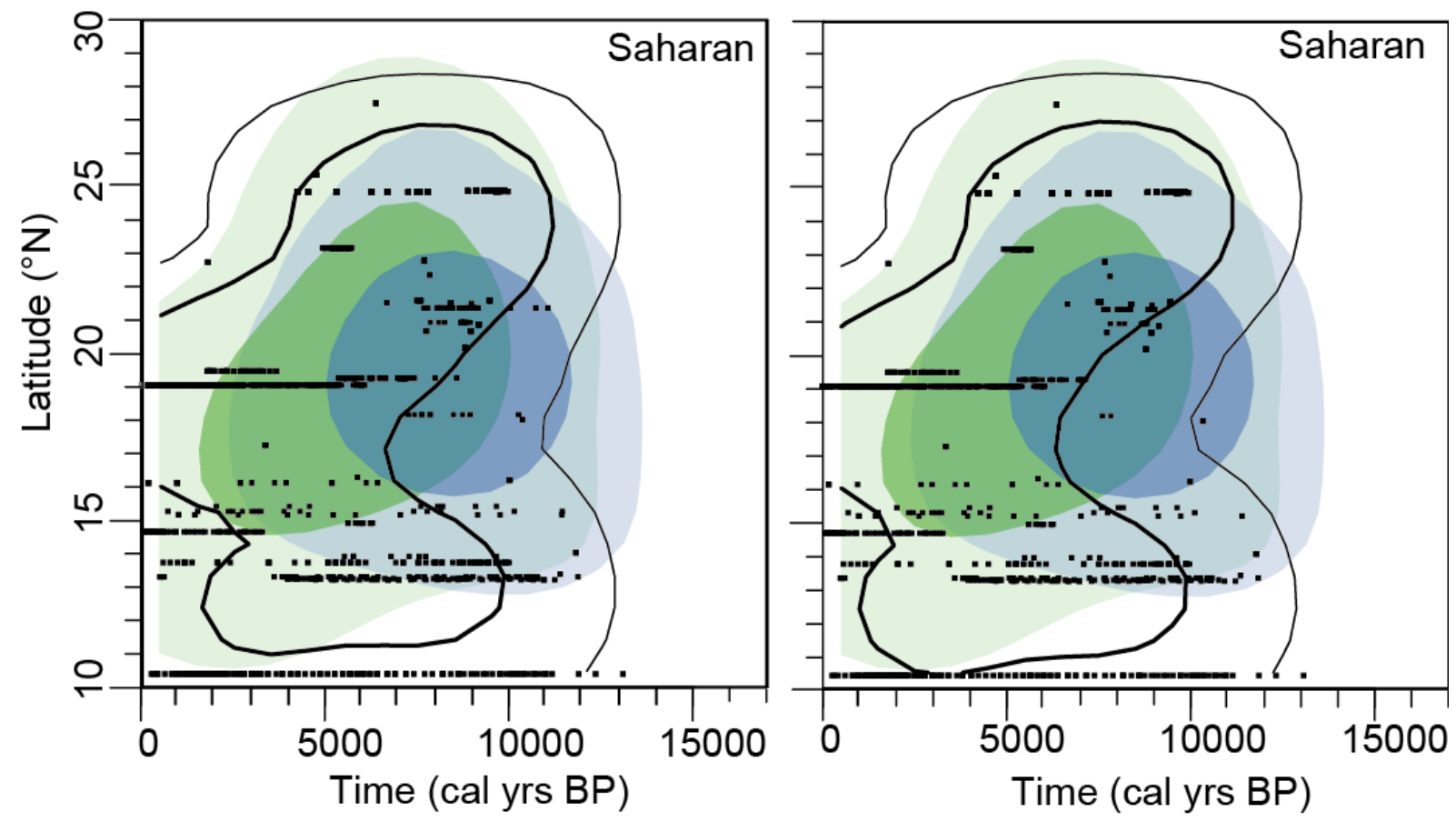

Figure S1. Spatio-temporal changes (in latitude and millennia) in pollen taxa presences within the Saharan group during the Holocene using probability density functions (Kühl et al., 2002). As for Figure 1 in the main text, the left panel reports spatio-temporal distribution based on non-exclusive taxa, while the right one reports distributions computed with only exclusive taxa. Bold line in each graph stands for the 0.5 isoprobability delineating the core zone in which $50 \%$ of the samples are the most concentrated (maximum presence). Similarly, thin line is the 0.85 isoprobability line delineating the maximum extent zone in which $85 \%$ of the samples are included (Lézine et al., 2011). Black dots are representative of pollen samples referenced in latitude and time. Blue and green probability density functions refer to lacustrine and palustrine distributions through time, respectively, with dark areas reflecting core zones ( 0.5 isoprobability computed on paleohydrological data), while light areas refer to the maximum extent (Lézine et al., 2011).

\section{References}

Ballouche, A., and Neumann, K.: A New Contribution to the Holocene Vegetation History of the WestAfrican Sahel - Pollen from Oursi, Burkina-Faso and Charcoal from 3 Sites in Northeast Nigeria, Vegetation History and Archaeobotany, 4, 31-39, 1995.

Ballouche, A., Reille, M., Thinon, M., Barakat, H. N., and Fontugne, M.: La végétation holocène des montagnes du Sahara Central: une nouvelle conception, 2e symposium de Palynologie africaine., Tervuren, Belgique, 1995, 9-17,

Ballouche, A.: Dynamique des paysages végétaux Sahélo-soudaniens et pratiques agro-pastorales à I'Holocène: exemples du Burkina Faso, Monographies, Bulletin de I'Association de Géographes Français, 2, 190-200, 1998.

Baumhauer, R., and Schulz, D.: The Holocene lake of Seguedine, Kaouar, NE Niger., Palaeoecology of Africa, 16, 283-290, 1984. 
Delibrias, G., Petit-Maire, N., and Schulz, E.: Les dépôts récents de la vallée du Shati, in: Le Shati, Lac Pleistocène du Fezzan (Libye), edited by: Petit-Maire, N., CNRS, Marseille, 86-88, 1982.

Guinet, P., and Planque, D.: Résultats de l'analyse pollinique in: Amekni, Néolithique ancien du Hoggar, edited by: G., C., Mémoires du Centre de Recherches anthropologiques préhistoriques et ethnographiques, Arts et métiers Graphiques, Organisme de Coopération scientifique en Algérie, Paris, 186-188, 1969.

Haynes, C. V., Eyles, C. H., Pavlish, L. A., Ritchie, J. C., and Rybak, M.: Holocene palaeoecology of the Eastern Sahara, Quaternary Science Reviews, 8, 109-136, 1989.

Jahns, S.: A Holocene pollen diagram from El Atrun, northern Sudan, Vegetation History and Archaeobotany, 4, 23-30, 1995.

Kröpelin, S., Verschuren, D., Lézine, A. M., Eggermont, H., Cocquyt, C., Francus, P., Cazet, J. P., Fagot, M., Rumes, B., Russell, J. M., Darius, F., Conley, D. J., Schuster, M., von Suchodoletz, H., and Engstrom, D. R.: Climate-driven ecosystem succession in the Sahara: The past 6000 years, Science, 320, 765-768, 2008.

Kühl, N., Gebhardt, C., Litt, T., and Hense, A.: Probability Density Functions as BotanicalClimatological TransferFunctions for Climate Reconstruction, Quaternary Research, 58, 381-392, doi:10.1006/qres.2002.2380, 2002.

Lézine, A.-M., Hély, C., Grenier, C., Braconnot, P., and Krinner, G.: Sahara and Sahel vulnerability to climate changes, lessons from Holocene hydrological data, Quaternary Science Reviews, 30, 30013012, 2011.

Lézine, A. M., Bieda, S., Faure, H., and Saos, J. L.: Etude palynologique et sédimentologique d'un milieu margino-littoral: la tourbière de Thiaye (Sénégal), Bulletin De La Societe Geologique De France, 38, 79-89, 1985.

Lézine, A. M.: Paléoenvironnements végétaux d'Afrique nord-tropicale depuis 12000 ans B.P. Analyse pollinique de séries sédimentaires continentales (Sénégal - Mauritanie). Volume I: Texte. Volume II : Annexes, Université Aix-Marseille 2 - Faculté des Sciences de Luminy (France), 207 pp., 1987.

Lézine, A. M.: Les variations de la couverture forestière mésophile d'Afrique occidentale au cours de I'Holocène, Comptes Rendus de l'Académie des Sciences, 307, 439-445, 1988a.

Lézine, A. M.: New pollen data from the Sahel, Senegal, Review of Palaeobotany and Palynology, 55, 141-154, 1988b.

Lézine, A. M., Casanova, J., and Hilaire-Marcel, C.: Across an early holocene humid phase in western Sahara, pollen and isotope stratigraphy, Geology, 18, 264-267, 1990.

Lézine, A. M., and Chateauneuf, J. J.: Peat in the Niayes of Senegal - Depositional Environment and Holocene Evolution, Journal of African Earth Sciences, 12, 171-179, 1991.

Lézine, A. M.: Chemchane, histoire d'une sebkha, Sécheresse, 4, 25-30, 1993.

Lézine, A. M.: Timing of vegetation changes at the end of the Holocene Humid Period at the Northern edge of the Atlantic and Indian monsoon systems, Comptes Rendus Geosciences, 341, 750-759, 2009.

Maley, J.: Etudes palynologiques dans le bassin du Tchad et paléoclimatologie de l'Afrique nordtropicale de 30000 ans à l'époque actuelle, Université des Sciences et Techniques du languedoc, Montpellier, 586, 1981.

Maley, J.: Le bassin du Tchad au quaternaire récent : formations sédimentaires, paléoenvironnements et préhistoire : la question des paléotchads., in: L'évolution de la végétation depuis deux millions d'années, edited by: Sémah, A. M. D., Renault-Miskovsky, J. d., Le Thomas, A. p., 
Cheddadi, R. c., Chepstow-Lusty, A. c., Jolly, D. c., Lebreton, V. c., (collab.), L. M. P., Maley, J. c., Scott, L. C., and (collab.), V. C. E., Guides de la Préhistoire Mondiale.Paléoenvironnements, Artcom et Errance, Paris, 179-217, 2004.

Medus, J., and Barbey, C.: Deux analyses polliniques de sédiments minéraux de Mauritanie méridionale, 75-79, 1979.

Mercuri, A. M., Trevisan Grandi, G., Mariotti Lippi, M., and Cremaschi, M.: New pollen data from the Uan Muhuggiag rockshelter (Libyan Sahara, VII-IV millennia BP). Wadi Teshuinat, in: Palaeoenvironment and prehistory in south-western Fezzan (Libyan Sahara). Survey and excavations in the Tadrart Acacus, Erg Uan Kasa, Messak Settafet and Edeyen of Murzuq, 1990-1995, edited by: Cremaschi, M., and di Lernia, S., All'Insegna del Giglio, Firenze, 107-122, 1998.

Mercuri, A. M.: Palynological analysis of the Early Holocene sequence in The Uan Afuda cave, Hunter-Gatherer societies of Central Sahara, in: Arid zone archaeology, edited by: Giglio, A. I. d., 149253, 1999.

Mercuri, A. M., and Trevisan Grandi, G.: Palynological analyses of the Late Pleistocene, Early Holocene and Middle Holocene layers. Uan Tabu in the settlement history of the Libyan Sahara. , Arid Zone Archaeology, Firenze, Italy, 2001, 237-251,

Ritchie, J. C., Eyles, C. H., and Haynes, C. V.: Sediment and pollen evidence for an early to midHolocene humid period in the eastern Sahara, Nature 314, 352-355, 1985.

Ritchie, J. C.: A holocene pollen record from Bir Atrun, Northwest Sudan, Pollen et Spores, 29, 391410, 1987.

Ritchie, J. C., and Haynes, C. V.: Holocene vegetation zonation in the eastern Sahara, Nature, 330, 645-647, 1987.

Ritchie, J. C.: Holocene pollen spectra from Oyo, northwestern Sudan: problems of interpretation in a hyperarid environment, The Holocene, 4, 9-15, 1994.

Salzmann, U.: Holocene vegetation history of Sahelian-zone of NE-Nigeria: Preliminary results, Palaeoecology of Africa, 24, 103-114, 1996.

Salzmann, U., and Waller, M.: The Holocene vegetational history of the Nigerian Sahel based on multiple pollen profiles, Review of Palaeobotany and Palynology, 100, 39-72, 1998.

Salzmann, U.: Zur holozänen vegetations- und klimaentwicklung der westafrikanischen savannen Paläoökologische untersuchungen in der Sahel- und Sudanzone NO-Nigerias, Ph.D., University of Wuerzburg, Frankfurt, 144 pp., 1999.

Salzmann, U.: Are modern savannas degraded forests ? - A Holocene pollen record from the Sudanian vegetation zone of NE Nigeria, Vegetation History and Archaeobotany, 9, 1-15, 2000.

Salzmann, U., Hoelzmann, P., and Morczinek, I.: Late Quaternary climate and vegetation of the Sudanian zone of northeast Nigeria, Quaternary Research, 58, 73-83, 2002.

Schulz, E.: Zur quartären Vegetationsgeschichte der zentralen Sahara unter Berücksichtigung eigener pollenanalystischer Untersuchungen aus dem Tibesti-Gebirge, Staatsexamensarbeit FU Berlin, 1973.

Schulz, E.: Zur Vegetation der östlichen zentralen Sahara und zu ihrer Entwicklung im Holozän., Würzburger Geographische Arbeiten, 1980.

Schulz, E., Pomel, S., Abichou, A., and Salzmann, U.: Climate and man. Questions and answers from both sides of the Sahara, 2e symposium de palynologie africaine/2nd Symposium on African Palynology, , Tervuren, Belgique, 1995, 35-47,

Thinon, M., Ballouche, A., and Reille, M.: Holocene vegetation of the Central Saharan Mount: The end of a myth, Holocene, 6, 457-462, 1996. 
139 Vincens, A., Lézine, A. M., Buchet, G., Lewden, D., Le Thomas, A., and Contributors: African pollen 140 database inventory of tree and shrub pollen types, Review of Palaeobotany and Palynology, 145, 141 135-141, 2007. 